\title{
Environmental Scanning Electron Microscopy for the Study of Gold Nanoparticle Uptake in Whole Cells
}

\author{
D. Peckys ${ }^{1}$ and N. de Jonge ${ }^{1,2}$ \\ ${ }^{1}$ INM Leibniz Institute for New Materials, Saarbrücken, Germany \\ ${ }^{2}$ Department of Molecular Physiology and Biophysics, Vanderbilt University School of \\ Medicine, Nashville, TN, USA
}

Research on cellular nanoparticle uptake, transport, storage, and excretion has attracted increasing interest during recent years. Imaging intracellular nanoparticles, however, still remains challenging, especially for quantitative measurements on a scale $<200 \mathrm{~nm}$. This is due to the diffraction limit of light microscopy on the one hand, and the limitations introduced by the sample preparation methods, such as ultrathin sectioning, required for electron microscopy (EM) on the other hand. Conventional EM studies providing quantitative data on nanoparticles in whole cells were so far only feasible for very small numbers of individual cells. In this study, we imaged intracellular gold nanoparticles (AuNPs) in a total of 176 whole, hydrated human lung cancer cells (A549). The focus of the study was to examine the influence of the size of the AuNPs $(10,15$, and $30 \mathrm{~nm})$ on their intracellular AuNP storage compartments, an effect that is still poorly understood [1].

A549 cells (from a human lung cancer cell line) were grown on microchips supporting thin electron transparent SiN windows. The cells were incubated with the AuNPs, chemically fixed, cooled to $3{ }^{\circ} \mathrm{C}$, and transferred into ultrapure water. Imaging was done with environmental scanning electron microscopy (ESEM). The microscope (FEI Quanta 400 FEG) was equipped with two detectors that were used synchronously: a scanning transmission electron microscopy (STEM) detector underneath the sample, and a gaseous secondary electron (GSE) detector mounted above the sample (Fig. 1a). In order to keep the cells in hydrated state during the imaging, the specimen chamber was filled with saturated water vapor, by keeping the temperature at $3{ }^{\circ} \mathrm{C}$ and the pressure around $740 \mathrm{~Pa}$. The presence of a thin water layer covering the cells was monitored with the GSE detector, whereas the STEM detector was used to record images of the intracellular AuNPs with high contrast.

Fig. 1b shows an image of a cell that was incubated for $2 \mathrm{~h}$ with serum protein coated AuNPs of $15 \mathrm{~nm}$ diameter. Afterwards, the cells were rinsed and incubated for a wait period of $22 \mathrm{~h}$ under cell culture growth conditions.. The image was recorded with a low magnification providing an orienting overview. A randomly-scattered distribution of dark round structures presumably vesicles filled with AuNPs can be seen. The dispersed character of the distribution was in contrast to earlier findings from similar AuNP uptake studies in COS7 cells (from a monkey kidney fibroblast cell line), where the majority of AuNP storage compartments had gathered in dense accumulations in proximity of the nucleus [2].

Fig. 1c was acquired at a higher magnification at the location indicated in Fig. 1b. The image shows that the vesicles contain AuNPs, and reveals a mostly uniform density of AuNPs within the vesicles. This distribution is in agreement with findings from COS7 cells [2] pointing to the adherence of AuNPs at the inner membrane of the vesicles. Fig 1d was recorded from a cell that had been incubated under similar conditions but with AuNPs of 30 $\mathrm{nm}$ diameter. At a first glance, the structures appear similar to those found in cells incubated with the smaller AuNPs. For a quantitative analysis of these vesicles, the sizes of $>1,000$ AuNP containing vesicles were measured. Subsequent analysis revealed that the vesicles 
filled with the $30 \mathrm{~nm}$-diameter AuNPs had on average 70-80 nm larger diameters than those filled with $15 \mathrm{~nm}$ AuNPs.

Our results show that the cellular compartments in which AuNPs are found can be influenced by the AuNPs themselves, namely by their size. This study exemplifies how STEM detection in ESEM can contribute to study nanoparticle routing mechanisms in statistically relevant amounts of intact cells.

\section{References:}

[1] T.-G. Iversen et al, Nano Today. 6 (2011), p. 176.

[2] D.B. Peckys \& N. de Jonge, Nano Lett. 11(4) (2011), p. 1733

[3] We thank M. Koch for help with the experiments, A. Kraegeloh for discussion and support of the experiments, and Protochips Inc, NC, USA for providing the microchips with silicon nitride support windows. We thank E. Arzt for his support through INM.
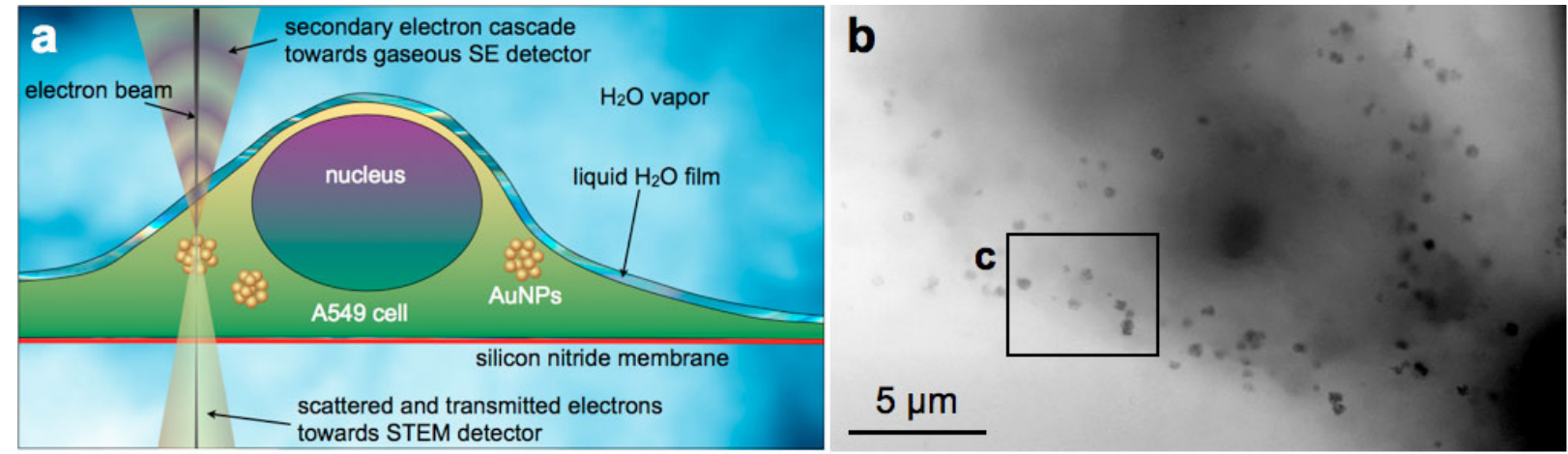

C

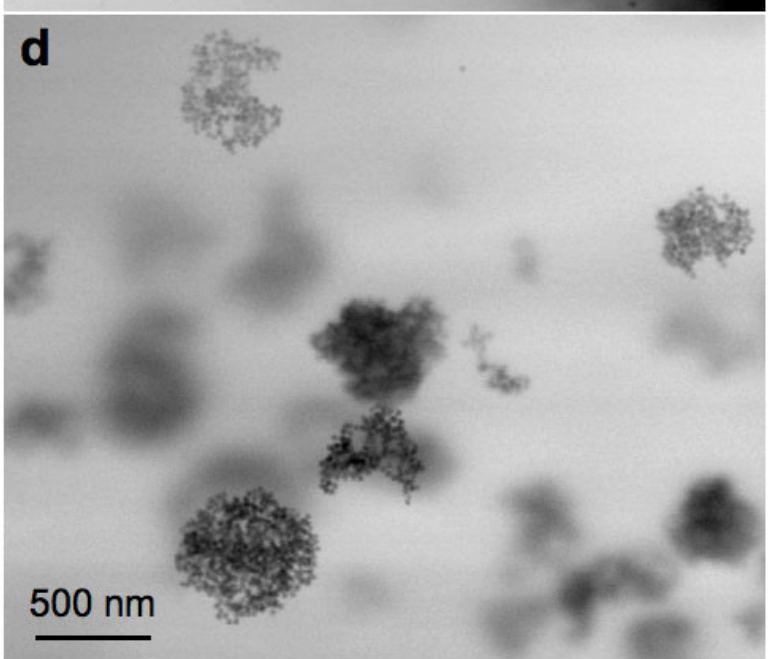

Figure 1. Whole A549 cells examined after uptake of $15 \mathrm{~nm}$ or $30 \mathrm{~nm}$ large AuNPs. a) Schematic representation of ESEM of whole cells in wet state. A focused electron beam was scanned over the cell, whereby a GSE detector, located above the sample, and a STEM detector, located beneath the sample, simultaneously collected the signals. b) STEM image showing an A549 cell after a $2 \mathrm{~h}$ incubation with serum coated $15 \mathrm{~nm}$ large AuNPs, followed by a $22 \mathrm{~h}$ waiting period. The magnification $(\mathrm{M})$ was $4,000 \times$. c) Image recorded at the location indicated with the c-labeled rectangle in $b . M=15,000 \times$. d) Image of cellular vesicles filled with serum coated AuNPs of $30 \mathrm{~nm}$ diameter after $2 \mathrm{~h}+22 \mathrm{~h}$ of incubation. M $=24,000 \times$. 